\title{
Teores de minerais em polpas e cascas de frutos de cultivares de bananeira
}

\author{
César Fernandes Aquino(1), Luiz Carlos Chamhum Salomão(2), Dalmo Lopes de Siqueira(2), \\ Paulo Roberto $\mathrm{Cecon}^{(3)}$ e Sônia Machado Rocha Ribeiro(4)
}

\begin{abstract}
(1)Universidade Federal de Viçosa (UFV), Avenida P.H. Rolfs, s/no, Campus Universitário, CEP 36570-900 Viçosa, MG, Brasil. E-mail: cesarfernandesaquino@yahoo.com.br ${ }^{(2)}$ UFV, Departamento deFitotecnia, Setorde Fruticultura.E-mail:Isalomao@ufv.br, siqueira@cead.ufv.br (3)UFV, Departamento de Estatística. E-mail: cecon@ufv.br ${ }^{(4)}$ UFV, Departamento de Nutrição e Saúde. E-mail: sribeiro@ufv.br
\end{abstract}

Resumo - O objetivo deste trabalho foi determinar a percentagem de matéria seca, o teor de minerais e a capacidade de fornecimento de minerais, com base na ingestão dietética de referência na polpa e na casca de frutos verdes e maduros de 15 cultivares de bananeira. Utilizaram-se quatro cachos por cultivar e seis frutos por unidade amostral. Foram coletadas amostras frescas da polpa e da casca, para digestões sulfúricas $(0,2 \mathrm{~g})$ e nitroperclóricas $(0,5 \mathrm{~g})$. Foram determinados: no extrato sulfúrico, o $\mathrm{N}$ total pelo método Kjeldahl; e, no extrato nitroperclórico, P, K, Ca, Mg, Cu, Mn, Fe, Zn e Se. O P foi determinado por colorimetria, e os demais nutrientes, por espectrofotometria de absorção atômica. O teor de minerais na polpa e na casca foi expresso com base na média dos dois estádios. Há diferenças entre cultivares de bananeira quanto à concentração de macro e micronutrientes na polpa e na casca, mas não entre frutos verdes e maduros. A cultivar Terrinha apresentou a maior percentagem de matéria seca na polpa, e as cultivares Marmelo e Maçã, na casca. Para N, P, $\mathrm{Fe}, \mathrm{Zn}$ e $\mathrm{Cu}$, a casca apresentou duas vezes o teor da polpa. O teor de $\mathrm{K}$ e $\mathrm{Mn}$ na casca foi de aproximadamente quatro vezes o da polpa. A polpa da cultivar Caipira (AAA) e a casca das cultivares Prata e Pacovan (AAB) apresentam os maiores teores de minerais.

Termos para indexação: Musa, composição mineral, composição nutricional, ingestão dietética de referência.

\section{Mineral content in the pulp and peel of banana cultivars}

\begin{abstract}
The objective of this work was to determine the dry matter percentage, the mineral content, and the ability to supply minerals, based on dietary reference intake in the pulp and peel of unripe and ripe fruits of 15 banana cultivars. Four bunches were used per cultivar, and six fruits per sample unit. Fresh pulp and peel samples were collected for sulfuric $(0.2 \mathrm{~g})$ and nitric perchloric $(0.5 \mathrm{~g})$ digestions. The following were determined: in the sulfuric extract, total $\mathrm{N}$ by the Kjeldahl method; and, in the nitro perchloric extract, $\mathrm{P}$, $\mathrm{K}, \mathrm{Ca}, \mathrm{Mg}, \mathrm{Cu}, \mathrm{Mn}, \mathrm{Fe}, \mathrm{Zn}$, and $\mathrm{Se} . \mathrm{P}$ was determined by colorimetry, and the other nutrients by atomic absorption spectrophotometry. The mineral content in the pulp and peel was expressed based on the average of the two stages. There are differences between banana cultivars regarding the concentration of macro- and micronutrients in the pulp and peel, but not between unripe and ripe fruits. The Terrinha cultivar showed the highest percentage of dry matter in the pulp, and the Marmelo and Maçã cultivars in the peel. For N, P, Fe, Zn, and $\mathrm{Cu}$, the peel showed twice the content of the pulp. The content of $\mathrm{K}$ and $\mathrm{Mn}$ in the peel was approximately four times that of the pulp. The pulp of the Caipira (AAA) cultivar, and the peel of the Prata and Pacovan (AAB) cultivars show the highest mineral contents.
\end{abstract}

Index terms: Musa, mineral composition, nutritional composition, dietary reference intake.

\section{Introdução}

A bananeira (Musa spp.) é uma das fruteiras mais cultivadas nos países de clima tropical e subtropical. A produção mundial de bananas e plátanos, em 2012, atingiu 139 milhões de toneladas, em aproximadamente 10,3 milhões de hectares colhidos. O Brasil ocupa o quinto lugar no ranking, com produção de 7,3 milhões de toneladas de banana e plátanos, em 481 mil hectares de área colhida (Food and Agriculture Organization of the United Nations, 2014).

Atualmente, há grande diversidade de cultivares de banana disponível no Brasil. Para algumas cultivares, como Pacovan, Prata Anã, Caipira, Nanicão, Maçã, Nanica e Prata Graúda, já são conhecidas as características agronômicas, as propriedades 
organolépticas e a composição química dos frutos (Gomes et al., 2007; Ramos et al., 2009).

Entretanto, em relação à quantificação de minerais e à percentagem de matéria seca na polpa e na casca, são escassas as informações sobre bananas cultivadas no Brasil. Além disso, quando disponíveis, referemse apenas ao subgrupo Cavendish, o que mostra a necessidade de trabalhos para avaliação dos demais subgrupos. Davey et al. (2009) avaliaram a polpa e a casca dos frutos de 47 genótipos de bananas e plátanos, provenientes de Camarões, de Uganda, dos Estados Unidos, das Filipinas e do Camboja, e observaram grande variação nos teores dos minerais, nos diferentes genótipos. Esses autores também relataram que alguns genótipos acumularam até três vezes mais o teor de minerais do que os outros. Davey et al. (2007), ao analisar a polpa e a casca de cinco cultivares de banana e plátanos em Camarões, constataram variações nos teores de minerais entre as cultivares e que os teores na casca foram superiores aos da polpa. Wall (2006) encontrou teores de matéria seca de 31,5 e $26,2 \%$, respectivamente, na polpa madura das cultivares Nanica e Williams, nos Estados Unidos. O mesmo autor concluiu que as duas cultivares estudadas podem ser consideradas boas fontes de $\mathrm{K}, \mathrm{Mg}, \mathrm{Cu}$ e $\mathrm{Mn}$ para a dieta humana, com base na ingestão diária de referência.

Os valores relatados para os minerais nas tabelas de composição alimentar frequentemente se baseiam em amostras de poucas ou de apenas uma cultivar; portanto, não são representativos do grande número de cultivares existente. Além disso, na maioria dos trabalhos, apenas a polpa é avaliada. A casca representa em torno de $40 \%$ do peso do fruto (Emaga et al., 2007) e é descartada quando a polpa é consumida; assim, uma porção considerável do fruto é jogada fora. A avaliação de diversas cultivares pode fornecer mais informações sobre o teor de minerais na banana, para indicação das cultivares que melhor satisfaçam a ingestão diária recomendada de minerais. Nesse sentido, a análise da casca pode servir de estímulo para a sua utilização na alimentação humana e animal.

Novas estratégias econômicas para aumentar a utilização da banana incluem: a produção de farinha de polpa e casca verdes e maduras (Alkarkhi et al., 2011); e a incorporação da farinha do fruto verde em vários produtos inovadores, como biscoitos lentamente digeríveis (Aparicio-Saguilan et al., 2007;
Agama-Acevedo et al., 2012), pão rico em fibras (Juarez-Garcia et al., 2006) e filmes comestíveis (Sothornvit \& Pitak, 2007). No entanto, muitos desses trabalhos avaliam apenas o teor de carboidratos e não a composição mineral desses frutos.

O objetivo deste trabalho foi determinar a percentagem de matéria seca, o teor de minerais e a capacidade de fornecimento de minerais, com base na ingestão dietética de referência, na polpa e na casca de frutos verdes e maduros de 15 cultivares de bananeira.

\section{Material e Métodos}

Cachos de 15 cultivares de bananeira (Tabela 1) foram colhidos de janeiro a abril de 2013, em plantas espaçadas de $3,5 \times 2,5 \mathrm{~m}$, em pomar experimental, com cinco anos de idade, na Universidade Federal de Viçosa (UFV), MG $\left(20^{\circ} 45^{\prime} \mathrm{S}, 42^{\circ} 51^{\prime} \mathrm{W}\right.$, a $650 \mathrm{~m}$ de altitude). O clima da região, segundo a classificação de Köppen, é do tipo Cwa, com médias anuais de temperatura, precipitação e umidade relativa do ar de $26,1^{\circ} \mathrm{C}, 1.340 \mathrm{~mm}$ e $80 \%$, respectivamente.

O bananal foi conduzido em regime de sequeiro, em Latossolo Vermelho-Amarelo, de relevo plano, textura média, e teores de $\mathrm{P}, \mathrm{K}, \mathrm{Zn}, \mathrm{Fe}, \mathrm{Cu}$ e Mn de 17,4, 54,0, 2,3, 111,7, 2,8 e 127,3 mg dm ${ }^{-3}$, respectivamente, e de $\mathrm{Ca}$ e $\mathrm{Mg}$ de 3,7 e 1,3 $\mathrm{cmol}_{\mathrm{c}}$ $\mathrm{dm}^{-3}$. As plantas foram submetidas a desfolhas e desbrotas periódicas; e anualmente, durante o período chuvoso (outubro a março), foram aplicados $500 \mathrm{~g}$ de superfosfato simples, $600 \mathrm{~g}$ de cloreto de potássio, $600 \mathrm{~g}$ de sulfato de amônio, $20 \mathrm{~g}$ de sulfato de zinco e $20 \mathrm{~g}$ de bórax, por família. As aplicações de cloreto de potássio e sulfato de amônio foram parceladas em três vezes. Não foram realizados feitos controles de pragas e doenças no pomar.

Os cachos foram colhidos aos primeiros sinais de amarelecimento nos frutos de cada cultivar e não foram pesados. A segunda, a terceira e a quarta penca de cada cacho foram retiradas e transportadas imediatamente ao Laboratório de Análises de Frutas, no campus da UFV, onde os frutos foram cortados rentes à almofada floral, tendo-se eliminado aqueles danificados, doentes e malformados. Em seguida, os frutos foram lavados em água de torneira e deixados em repouso sobre papel absorvente por alguns minutos, para coagulação do látex. Foram selecionados 12 frutos no estádio 1, com coloração da casca verde-escura (Dadzie \& Orchard, 1997), sendo seis deles decompostos em polpa e casca. 
Os outros seis frutos foram imersos em solução de ethephon $\left(1,2 \mathrm{~g} \mathrm{~L}^{-1}\right)$ por $8 \mathrm{~min}$, para uniformizar o amadurecimento. Após secarem por $15 \mathrm{~min}$, os frutos foram mergulhados em solução fungicida de Procloraz $\left(0,22 \mathrm{~g} \mathrm{~L}^{-1}\right)$ por $5 \mathrm{~min}$. Decorrido esse tempo, os seis frutos foram acondicionados em caixas de plástico e mantidos em temperatura ambiente até atingirem o estádio de cor 6, com casca completamente amarela (Dadzie \& Orchard, 1997).

Os seis frutos verdes (polpa e casca) não receberam nenhum tratamento químico e, após limpos, foram processados, com retirada de amostras para determinação da massa de matéria seca e para quantificação dos minerais. As amostras compostas foram acondicionadas em envoltório de alumínio e pesadas em balança semianalítica, identificadas, submetidas ao congelamento em nitrogênio líquido e acondicionadas em ultrafreezer a $-80^{\circ} \mathrm{C}$, até o momento das digestões. O mesmo procedimento foi adotado para os seis frutos, no estádio 6 .

Utilizou-se o delineamento inteiramente casualizado, com 15 tratamentos (cultivares), quatro repetições (cachos) e seis frutos por unidade amostral. Para cada cultivar, analisaram-se polpa e casca verdes e maduras. Os resultados referentes a cada parte do fruto, polpa e casca, foram avaliados como ensaios distintos.

A percentagem de matéria seca da polpa e da casca de frutos verdes e maduros foi determinada gravimetricamente. Para isso, amostras compostas de $20 \mathrm{~g}$ da polpa e da casca foram secas em estufa com

Tabela 1. Grupo genômico e subgrupo das 15 cultivares de bananeira (Musa spp.) avaliadas.

\begin{tabular}{lcc}
\hline Cultivares & Grupo genômico & Subgrupo \\
\hline Ouro & AA & - \\
Nanica & AAA & Cavendish \\
Nanicão & AAA & Cavendish \\
Caru-Roxa & AAA & - \\
Caru-Verde & AAA & - \\
Caipira & AAA & - \\
Prata & AAB & Prata \\
Prata Anã & AAB & Prata \\
Maçã & AAB & - \\
Mysore & AAB & - \\
Pacovan & AAB & Prata \\
Terrinha & AAB & Terra \\
Marmelo & ABB & - \\
Prata Graúda (SH-3640) & AAAB & - \\
Caju & Não definido & - \\
\hline
\end{tabular}

circulação forçada de ar, a $70^{\circ} \mathrm{C}$, até peso constante e novamente pesadas.

Para as análises de minerais, foram coletadas amostras frescas da polpa e da casca, para digestões sulfúricas $(0,2 \mathrm{~g})$ e nitroperclóricas $(0,5 \mathrm{~g})$. No extrato sulfúrico, determinou-se o $\mathrm{N}$ total pelo método Kjeldahl (Helrich, 1990), e, no extrato nitroperclórico, quantificou-se $\mathrm{P}, \mathrm{K}, \mathrm{Ca}, \mathrm{Mg}, \mathrm{Cu}, \mathrm{Mn}, \mathrm{Fe}, \mathrm{Zn}$ e Se. O P foi determinado por colorimetria, conforme Braga \& Defelipo (1974), e os demais nutrientes foram quantificados por espectrofotometria de absorção atômica, em espectrofotômetro, modelo SpectrAA 220 FS (Varian Medical Systems, Belrose, Austrália), nas condições instrumentais recomendadas. Foram utilizados padrões Merck (Merck Brasil, Rio de Janeiro, RJ) para preparo das curvas-padrão de: $\mathrm{K}$ e $\mathrm{Ca}$, em concentração na faixa de 0 a $20 \mathrm{mg} \mathrm{L}^{-1} ; \mathrm{Mg}$, $\mathrm{Zn}$ e $\mathrm{Cu}$, na faixa de 0 a $2 \mathrm{mg} \mathrm{L}^{-1}$; e Fe e $\mathrm{Mn}$, na faixa de 0 a $15 \mathrm{mg} \mathrm{L}^{-1}$; os resultados foram expressos em $\mathrm{g} \mathrm{kg}^{-1}$ e mg kg-1 para macro e micronutrientes, respectivamente, com base na percentagem de matéria seca.

Os dados referentes aos teores de macro e micronutrientes na polpa e na casca foram expressos com base na média dos resultados obtidos nos frutos verdes e maduros. A quantidade de nutrientes exportada foi calculada apenas para os frutos verdes, a partir da matéria fresca. A percentagem de minerais das partes do fruto foi calculada somente para as cultivares que se destacaram com maior teor na polpa ou na casca, com base na percentagem de matéria fresca.

Os dados das variáveis referentes à comparação entre cultivares foram submetidos à análise de variância, e as médias foram agrupadas pelo teste de Scott \& Knott, a $1 \%$ de probabilidade, tendo-se utilizado o programa SAEG 9.1 (Saeg, 2007).

As cultivares foram ranqueadas quanto ao teor dos minerais avaliados. Para tanto, na polpa e na casca, atribuiu-se uma nota para cada mineral, a partir do teste de média de Scott \& Knott, em que as letras correspondentes a cada média foram utilizadas para ranquear as cultivares: $\mathrm{a}=1, \mathrm{~b}=2, \mathrm{c}=3, \mathrm{~d}=4$, e assim sucessivamente. Dessa forma, para a polpa e a casca de cada cultivar, foi calculado o somatório das notas de todos os minerais. Esse somatório representou a nota geral, a qual foi usada no ranqueamento das cultivares, sendo que as mais ricas em minerais receberam as menores notas. 
A capacidade de fornecimento de minerais a partir da ingestão dietética de referência (IDR) foi calculada para o consumo de $100 \mathrm{~g}$ da polpa ou da casca, com base na matéria fresca, tendo-se calculado o fornecimento para a cultivar com o maior teor de minerais, em comparação às outras três cultivares mais difundidas no Brasil. Os cálculos foram realizados apenas com base na IDR para homens adultos (National Research Council, 2001, 2004).

\section{Resultados e Discussão}

A percentagem de matéria seca na polpa foi, em média, o dobro da obtida na casca, em razão da alta concentração de carboidratos na polpa, que pode atingir entre 20 e $25 \%$ da matéria fresca (Emaga et al., 2007). A maior percentagem de matéria seca na polpa foi encontrada para a cultivar Terrinha (AAB), enquanto as menores percentagens foram verificadas para as cultivares Nanica, Caru-Roxa, Caru-Verde, Caipira (AAA) e Prata Graúda (AAAB). Wall (2006) obteve percentagem de matéria seca na polpa madura das cultivares Nanica e Williams de 31,5 e 26,2\%, respectivamente, semelhante ao observado para a maioria das cultivares avaliadas. Na casca, as cultivares Marmelo (ABB) e Maçã (AAB) se destacaram com os maiores percentuais (Tabela 2).

Não houve variação significativa entre os teores de minerais nos frutos verdes e maduros, assim, os dados referentes aos macro e aos micronutrientes representam a média dos dois estádios. Em relação aos teores médios de minerais, constatou-se que, para $\mathrm{N}, \mathrm{P}, \mathrm{Fe}, \mathrm{Zn}$ e $\mathrm{Cu}$, a casca apresentou o dobro do teor observado na polpa. Os teores médios de K e Mn na casca foram, aproximadamente, o quádruplo dos teores na polpa. O Se não foi detectado em nenhuma parte avaliada.

De acordo com o critério proposto para o ranqueamento das cultivares, a ordem decrescente para o teor de macro e micronutrientes na polpa é a seguinte: Caipira $>$ Caru-Roxa $>$ Caru-Verde $>$ Mysore $>$ Nanica $=$ Prata Graúda $>$ Caju $>$ Maçã $>$ Nanicão, Pacovan $>$ Prata Anã $>$ Marmelo $>$ Prata $=$ Terrinha $>$ Ouro (Tabela 3), o que mostra que Caipira (AAA) e Ouro (AA) são, respectivamente, as cultivares avaliadas com maior e menor teor global de minerais na polpa.

$\mathrm{Na}$ polpa, a cultivar Nanica (AAA) apresentou maior teor de N. Para K, as cultivares Caru-Roxa
(AAA), Caru-Verde (AAA) e Caipira (AAA) apresentaram os maiores teores. Já para $\mathrm{Mg}$, as cultivares Maçã (AAB), Mysore (AAB) e Pacovan (AAB) se destacaram. Os minerais $\mathrm{P}$ e $\mathrm{Ca}$ foram maiores na polpa da cultivar Caipira, enquanto o teor de Mn apresentou ampla predominância na cultivar Caju, com teor aproximadamente 17 vezes maior que o da Nanica. Para o teor de $\mathrm{Cu}$, não houve diferença entre as cultivares, com exceção de Nanicão, Terrinha e Marmelo que apresentaram menor teor (Tabela 3). Davey et al. (2009), ao avaliar os frutos nos estádios de cor 1 a 7, em 47 genótipos de bananas e plátanos, provenientes de Camarões, da Uganda, dos Estados Unidos, das Filipinas e do Camboja, observaram teores médios de macro e micronutrientes semelhantes aos obtidos no presente trabalho.

De acordo com o critério proposto para ranqueamento das cultivares, a ordem decrescente do teor de macro e micronutrientes na casca é a seguinte: Prata $=$ Pacovan $>$ Mysore $=$ Caipira $>$ Prata Anã $>$ Ouro $>$ Prata Graúda $>$ Caru-Roxa $=$ Nanica $>$ Terrinha $>$ Nanicão $=$ Caru-Verde $>$ Marmelo $>$ Maçã (Tabela 4). Entre as cultivares avaliadas, Prata (AAB) e Pacovan (AAB) apresentaram os maiores teores de minerais na casca, enquanto Maçã (AAB) é a mais pobre em minerais.

Tabela 2. Percentagem de matéria seca da polpa e da casca de frutos verdes e maduros de 15 cultivares de bananeira (Musa spp. $^{(1)}$.

\begin{tabular}{lcc}
\hline Cultivares & \multicolumn{2}{c}{ Matéria seca média de frutos (\%) } \\
\cline { 2 - 3 } & Polpa & Casca \\
\hline Ouro (AA) & $32,75 \mathrm{~b}$ & $15,19 \mathrm{~b}$ \\
Nanica (AAA) & $25,69 \mathrm{e}$ & $12,25 \mathrm{c}$ \\
Nanicão (AAA) & $27,81 \mathrm{~d}$ & $13,81 \mathrm{~b}$ \\
Caru-Roxa (AAA) & $24,12 \mathrm{e}$ & $13,88 \mathrm{~b}$ \\
Caru-Verde (AAA) & $26,19 \mathrm{e}$ & $14,75 \mathrm{~b}$ \\
Caipira (AAA) & $25,31 \mathrm{e}$ & $11,81 \mathrm{c}$ \\
Prata (AAB) & $30,50 \mathrm{c}$ & $12,69 \mathrm{c}$ \\
Prata Anã (AAB) & $31,63 \mathrm{~b}$ & $13,13 \mathrm{c}$ \\
Maçã (AAB) & $29,94 \mathrm{c}$ & $17,13 \mathrm{a}$ \\
Mysore (AAB) & $28,31 \mathrm{~d}$ & $14,31 \mathrm{~b}$ \\
Pacovan (AAB) & $32,56 \mathrm{~b}$ & $13,44 \mathrm{~b}$ \\
Terrinha (AAB) & $38,38 \mathrm{a}$ & $13,74 \mathrm{~b}$ \\
Marmelo (ABB) & $33,31 \mathrm{~b}$ & $18,44 \mathrm{a}$ \\
Prata Graúda (SH-3640) (AAAB) & $24,94 \mathrm{e}$ & $11,81 \mathrm{c}$ \\
Caju & $28,69 \mathrm{~d}$ & $14,81 \mathrm{~b}$ \\
\hline Média & 29,34 & 14,08 \\
CV (\%) & 4,94 & 9,30 \\
\hline
\end{tabular}

(1)Médias seguidas de letras iguais, nas colunas, não diferem pelo teste de Scott \& Knott, a $1 \%$ de probabilidade. 
$\mathrm{Na}$ casca, os maiores teores de $\mathrm{K}$ e $\mathrm{Mg}$ foram obtidos nas cultivares Ouro (AA) e Pacovan (AAB), respectivamente. Semelhantemente ao observado na polpa, a cultivar Caju apresentou teor de $\mathrm{Mn}$ aproximadamente 6,5 vezes maior que o da Nanica. Para os demais minerais, os teores variaram entre as cultivares (Tabela 4). Kalemelawa et al. (2012) realizaram a quantificação de minerais na casca de banana madura, em cultivar não informada, e observaram maior teor de $\mathrm{K}\left(66,40 \mathrm{~g} \mathrm{~kg}^{-1}\right)$, teores de $\mathrm{Ca}\left(2,11 \mathrm{~g} \mathrm{~kg}^{-1}\right)$ e $\mathrm{Mg}\left(1,02 \mathrm{~g} \mathrm{~kg}^{-1}\right)$ próximos, e teores de P $\left(0,22 \mathrm{~g} \mathrm{~kg}^{-1}\right)$ e $\mathrm{N}\left(10,5 \mathrm{~g} \mathrm{~kg}^{-1}\right)$ bastante inferiores aos obtidos no presente trabalho. Emaga et al. (2007) relataram teores de K (47 a $63 \mathrm{~g} \mathrm{~kg}^{-1}$ ) e

Tabela 3. Teores de macro e micronutrientes na polpa dos frutos de 15 cultivares de bananeira (Musa spp. $)^{(1)}$.

\begin{tabular}{|c|c|c|c|c|c|c|c|c|c|c|}
\hline \multirow[t]{2}{*}{ Cultivares } & \multicolumn{5}{|c|}{ Macronutrientes ( $\mathrm{g} \mathrm{kg}^{-1}$ de matéria seca) } & \multicolumn{4}{|c|}{ Micronutrientes ( $\mathrm{mg} \mathrm{kg}^{-1}$ de matéria seca) } & \multirow[t]{2}{*}{ Ranqueamento $^{(2)}$} \\
\hline & $\mathrm{N}$ & $\mathrm{P}$ & $\mathrm{K}$ & $\mathrm{Mg}$ & $\mathrm{Ca}$ & $\mathrm{Fe}$ & $\mathrm{Zn}$ & $\mathrm{Mn}$ & $\mathrm{Cu}$ & \\
\hline Ouro & $9,86 \mathrm{c}$ & $2,43 \mathrm{e}$ & $7,83 \mathrm{~d}$ & $0,72 \mathrm{~d}$ & $0,09 \mathrm{e}$ & $28,10 \mathrm{~b}$ & $21,88 \mathrm{~b}$ & $4,01 \mathrm{e}$ & $4,52 \mathrm{a}$ & 31 \\
\hline Nanica & $13,50 \mathrm{a}$ & $3,44 \mathrm{c}$ & $9,94 b$ & $0,98 \mathrm{~b}$ & $0,14 d$ & $35,86 a$ & $22,96 b$ & $3,53 \mathrm{e}$ & $4,31 \mathrm{a}$ & 21 \\
\hline Nanicão & $12,10 \mathrm{~b}$ & $2,97 \mathrm{~d}$ & $8,40 \mathrm{c}$ & $0,94 \mathrm{~b}$ & $0,13 \mathrm{~d}$ & $32,15 \mathrm{a}$ & $23,20 \mathrm{~b}$ & $5,48 \mathrm{e}$ & $3,41 b$ & 25 \\
\hline Caru-Roxa & $10,88 \mathrm{~b}$ & $3,34 \mathrm{c}$ & $11,80 \mathrm{a}$ & $0,95 b$ & $0,24 \mathrm{~b}$ & $34,14 a$ & $30,05 \mathrm{a}$ & $9,72 d$ & $5,14 \mathrm{a}$ & 17 \\
\hline Caru-Verde & $11,50 \mathrm{~b}$ & $3,31 \mathrm{c}$ & $11,19 a$ & $1,00 \mathrm{~b}$ & $0,18 \mathrm{c}$ & $30,85 a$ & $25,73 b$ & $12,30 \mathrm{c}$ & $4,36 \mathrm{a}$ & 18 \\
\hline Caipira & $11,88 \mathrm{~b}$ & $5,25 \mathrm{a}$ & $11,27 \mathrm{a}$ & $0,90 \mathrm{c}$ & $0,28 \mathrm{a}$ & $30,77 \mathrm{a}$ & $20,73 \mathrm{c}$ & $18,88 \mathrm{~b}$ & $5,55 \mathrm{a}$ & 15 \\
\hline Prata & $8,97 \mathrm{c}$ & $2,16 \mathrm{e}$ & $7,29 \mathrm{~d}$ & $0,85 \mathrm{c}$ & $0,15 d$ & $30,38 \mathrm{a}$ & $20,55 \mathrm{c}$ & $5,75 \mathrm{e}$ & $4,26 \mathrm{a}$ & 29 \\
\hline Prata Anã & $9,22 \mathrm{c}$ & $2,71 d$ & $7,18 \mathrm{~d}$ & $0,89 \mathrm{c}$ & $0,20 \mathrm{c}$ & $28,82 \mathrm{~b}$ & $22,14 b$ & $8,39 d$ & $4,87 \mathrm{a}$ & 26 \\
\hline Maçã & $9,49 \mathrm{c}$ & $4,48 b$ & $8,68 \mathrm{c}$ & $1,13 a$ & $0,13 d$ & $26,56 \mathrm{~b}$ & $19,38 \mathrm{c}$ & $5,85 \mathrm{e}$ & $4,13 \mathrm{a}$ & 24 \\
\hline Mysore & $10,18 \mathrm{c}$ & $3,62 \mathrm{c}$ & $9,50 \mathrm{~b}$ & $1,06 a$ & $0,25 b$ & $32,11 \mathrm{a}$ & $20,36 \mathrm{c}$ & $11,26 \mathrm{c}$ & $4,15 \mathrm{a}$ & 19 \\
\hline Pacovan & $9,44 \mathrm{c}$ & $2,75 d$ & $7,96 \mathrm{~d}$ & $1,05 \mathrm{a}$ & $0,16 \mathrm{~d}$ & $29,25 b$ & $23,11 b$ & $7,46 \mathrm{~d}$ & $4,40 \mathrm{a}$ & 25 \\
\hline Terrinha & $9,63 \mathrm{c}$ & $2,63 d$ & $6,57 \mathrm{~d}$ & $0,74 d$ & $0,08 \mathrm{e}$ & $26,49 b$ & $17,44 \mathrm{c}$ & $17,84 b$ & $2,74 b$ & 29 \\
\hline Marmelo & $7,90 \mathrm{c}$ & $3,48 \mathrm{c}$ & $8,89 \mathrm{c}$ & $0,87 \mathrm{c}$ & $0,12 d$ & $26,29 b$ & $19,05 \mathrm{c}$ & $4,80 \mathrm{e}$ & $3,54 b$ & 28 \\
\hline Prata Graúda & $12,34 b$ & $2,81 \mathrm{~d}$ & $9,10 \mathrm{c}$ & $0,97 \mathrm{~b}$ & $0,18 \mathrm{c}$ & $33,18 \mathrm{a}$ & $24,62 b$ & $14,74 \mathrm{c}$ & $3,93 \mathrm{a}$ & 21 \\
\hline Caju & $9,21 \mathrm{c}$ & $3,39 \mathrm{c}$ & $9,83 \mathrm{~b}$ & $0,79 \mathrm{~d}$ & $0,19 \mathrm{c}$ & $27,42 b$ & $16,34 \mathrm{c}$ & $61,00 \mathrm{a}$ & $4,46 \mathrm{a}$ & 21 \\
\hline Média & 10,40 & 3,28 & 9,02 & 0,91 & 0,16 & 30,15 & 21,83 & 12,73 & 4,25 & - \\
\hline CV (\%) & 7,27 & 6,95 & 8,48 & 6,02 & 9,92 & 7,75 & 11,22 & 16,69 & 14,20 & - \\
\hline
\end{tabular}

${ }^{(1)}$ Médias seguidas de letras iguais, nas colunas, não diferem pelo teste de Scott $\&$ Knott, a $1 \%$ de probabilidade. ${ }^{(2)}$ Ranqueamento das cultivares por nota quanto aos teores de minerais, em que as letras correspondem a cada média: $\mathrm{a}=1, \mathrm{~b}=2, \mathrm{c}=3, \mathrm{~d}=4$, e assim sucessivamente para cálculo da nota geral.

Tabela 4. Teores de macro e micronutrientes na casca dos frutos de 15 cultivares de bananeira (Musa spp. $)^{(1)}$.

\begin{tabular}{|c|c|c|c|c|c|c|c|c|c|c|}
\hline \multirow[t]{2}{*}{ Cultivares } & \multicolumn{5}{|c|}{ Macronutrientes ( $\mathrm{g} \mathrm{kg}^{-1}$ de matéria seca) } & \multicolumn{4}{|c|}{ Micronutrientes ( $\mathrm{mg} \mathrm{kg}^{-1}$ de matéria seca) } & \multirow[t]{2}{*}{ Ranqueamento $^{(2)}$} \\
\hline & $\mathrm{N}$ & $\mathrm{P}$ & $\mathrm{K}$ & $\mathrm{Mg}$ & $\mathrm{Ca}$ & $\mathrm{Fe}$ & $\mathrm{Zn}$ & $\mathrm{Mn}$ & $\mathrm{Cu}$ & \\
\hline Ouro & $22,75 a$ & $6,82 b$ & $51,47 \mathrm{a}$ & $1,20 \mathrm{~d}$ & $1,98 \mathrm{~b}$ & $67,38 \mathrm{a}$ & $56,57 \mathrm{a}$ & $33,62 \mathrm{e}$ & $8,86 a$ & 19 \\
\hline Nanica & $25,32 \mathrm{a}$ & $6,22 b$ & $40,96 b$ & $0,83 \mathrm{e}$ & $1,25 \mathrm{c}$ & $77,05 \mathrm{a}$ & $56,55 \mathrm{a}$ & $17,15 \mathrm{f}$ & $7,18 b$ & 23 \\
\hline Nanicão & $20,28 b$ & $4,85 \mathrm{c}$ & $40,34 b$ & $1,17 \mathrm{~d}$ & $1,62 \mathrm{c}$ & $67,77 \mathrm{a}$ & $55,65 \mathrm{a}$ & $23,89 f$ & $5,22 \mathrm{c}$ & 25 \\
\hline Caru-Roxa & $16,10 \mathrm{~b}$ & $7,81 \mathrm{a}$ & $30,38 \mathrm{c}$ & $1,19 \mathrm{~d}$ & $1,95 b$ & $56,87 \mathrm{~b}$ & $42,84 b$ & $37,76 \mathrm{e}$ & $7,49 b$ & 23 \\
\hline Caru-Verde & $16,50 \mathrm{~b}$ & $6,81 \mathrm{~b}$ & $31,89 \mathrm{c}$ & $0,94 \mathrm{e}$ & $1,68 \mathrm{c}$ & $56,00 \mathrm{~b}$ & $46,18 b$ & $52,76 \mathrm{c}$ & $6,06 \mathrm{c}$ & 25 \\
\hline Caipira & $18,87 \mathrm{~b}$ & $7,37 \mathrm{a}$ & $44,91 \mathrm{~b}$ & $1,10 \mathrm{~d}$ & $2,19 b$ & $66,40 \mathrm{a}$ & $56,11 \mathrm{a}$ & $93,60 \mathrm{~b}$ & $10,21 \mathrm{a}$ & 16 \\
\hline Prata & $22,69 a$ & $8,35 \mathrm{a}$ & $35,79 \mathrm{c}$ & $1,58 \mathrm{~b}$ & $2,84 a$ & $68,52 \mathrm{a}$ & $64,17 \mathrm{a}$ & $55,36 \mathrm{c}$ & $7,43 b$ & 14 \\
\hline Prata Anã & $23,18 \mathrm{a}$ & $4,76 \mathrm{c}$ & $30,90 \mathrm{c}$ & $1,67 b$ & $2,24 b$ & $65,46 a$ & $59,48 \mathrm{a}$ & $53,67 \mathrm{c}$ & $9,72 \mathrm{a}$ & 19 \\
\hline Maçã & $19,27 b$ & $4,87 \mathrm{c}$ & $32,94 \mathrm{c}$ & $1,02 \mathrm{~d}$ & $1,50 \mathrm{c}$ & $48,32 b$ & $40,52 b$ & $21,10 \mathrm{f}$ & $4,80 \mathrm{c}$ & 28 \\
\hline Mysore & $19,50 \mathrm{~b}$ & $6,55 b$ & $41,39 b$ & $1,73 b$ & $2,41 \mathrm{a}$ & $71,14 a$ & $50,28 \mathrm{a}$ & $55,39 \mathrm{c}$ & $7,68 b$ & 16 \\
\hline Pacovan & $21,85 \mathrm{a}$ & $6,35 b$ & $34,99 \mathrm{c}$ & $2,09 a$ & $2,55 \mathrm{a}$ & $66,85 \mathrm{a}$ & $55,76 \mathrm{a}$ & $58,69 \mathrm{c}$ & $7,83 b$ & 15 \\
\hline Terrinha & $26,55 \mathrm{a}$ & $4,97 \mathrm{c}$ & $39,83 \mathrm{~b}$ & $0,86 \mathrm{e}$ & $0,92 \mathrm{~d}$ & $64,35 \mathrm{a}$ & $57,88 \mathrm{a}$ & $41,68 d$ & $6,16 \mathrm{c}$ & 24 \\
\hline Marmelo & $16,54 b$ & $8,59 a$ & $22,95 \mathrm{c}$ & $1,04 d$ & $0,81 \mathrm{~d}$ & $45,31 \mathrm{~b}$ & $43,99 b$ & $24,10 \mathrm{f}$ & $4,93 \mathrm{c}$ & 27 \\
\hline Prata Graúda & $26,12 \mathrm{a}$ & $4,51 \mathrm{c}$ & $27,27 \mathrm{c}$ & $1,14 \mathrm{c}$ & $1,61 \mathrm{c}$ & $72,03 \mathrm{a}$ & $53,41 \mathrm{a}$ & $56,06 \mathrm{c}$ & $7,51 b$ & 20 \\
\hline Caju & $15,84 \mathrm{~b}$ & $6,52 b$ & $31,46 \mathrm{c}$ & $0,73 \mathrm{e}$ & $2,23 b$ & $48,60 \mathrm{~b}$ & $38,60 \mathrm{~b}$ & $111,37 \mathrm{a}$ & $8,53 \mathrm{a}$ & 20 \\
\hline Média & 20,75 & 6,35 & 35,83 & 1,22 & 1,85 & 62,80 & 51,86 & 49,08 & 7,30 & - \\
\hline CV (\%) & 12,19 & 9,15 & 12,44 & 10,09 & 14,18 & 10,79 & 11,98 & 12,92 & 15,41 & - \\
\hline
\end{tabular}

${ }^{(1)}$ Médias seguidas de letras iguais, nas colunas, não diferem pelo teste de Scott \& Knott, a $1 \%$ de probabilidade. ${ }^{(2)}$ Ranqueamento das cultivares por nota quanto aos teores de minerais, em que as letras correspondem a cada média: $\mathrm{a}=1, \mathrm{~b}=2, \mathrm{c}=3, \mathrm{~d}=4$, e assim sucessivamente para cálculo da nota geral. 
$\mathrm{Ca}\left(1,5\right.$ a $\left.7,2 \mathrm{~g} \mathrm{~kg}^{-1}\right)$ superiores. Entretanto, para $\mathrm{P}$, $\mathrm{Mg}, \mathrm{Fe}, \mathrm{Zn}, \mathrm{Cu}$ e $\mathrm{Mn}$, esses autores obtiveram teores bastante inferiores. Gondim et al. (2005), ao estudar a composição mineral das cascas de sete frutas maduras, incluindo a banana, cuja cultivar não foi informada, obtiveram teores de $\mathrm{K}, \mathrm{Ca}, \mathrm{Mg}, \mathrm{Fe}, \mathrm{Zn}$ e $\mathrm{Cu}$ bastante inferiores aos do presente trabalho.

A composição mineral da banana pode refletir o conteúdo mineral dos solos e varia de acordo com o clima das diferentes regiões de cultivo, a maturidade do fruto e as práticas culturais (Emaga et al., 2007). Isso pode explicar as variações observadas entre os diferentes trabalhos citados. No entanto, para plantas cultivadas sob as mesmas condições edafoclimáticas e de adubação, as variações entre os teores de minerais são atribuídas às particularidades intrínsecas a cada cultivar (Davey et al., 2007), uma vez que algumas são capazes de acumular teores até três vezes mais elevados de determinado mineral em seus frutos sob as mesmas condições de cultivo (Davey et al., 2009).

$\mathrm{K}$ e $\mathrm{N}$ foram os macronutrientes mais absorvidos e exportados pelas bananeiras, seguidos por $\mathrm{Mg}, \mathrm{Ca}$ e $\mathrm{P}$ (Tabela 5). Soares et al. (2008) também observaram que $\mathrm{K}$ e N são os macronutrientes mais exportados, mas que houve menor exportação de $\mathrm{Mg}$. Os micronutrientes Fe e $\mathrm{Zn}$ foram os mais acumulados nos frutos, seguidos por $\mathrm{Mn}$ e Cu. Os teores médios de minerais exportados pelas
15 cultivares avaliadas são maiores do que os relatados por Teixeira et al. (2008), ao analisar a exportação de minerais em cachos (frutos + engaço), com exceção de K. Hoffmann et al. (2010) verificaram a exportação de maiores teores de $\mathrm{Mn}$ em comparação aos de $\mathrm{Fe}$ e Zn. Essas diferenças podem ser explicadas pela disponibilidade dos nutrientes no solo, nas diferentes regiões onde as bananeiras foram cultivadas.

Segundo o critério de ranqueamento de cultivares, a ordem decrescente de exportação de macro e micronutrientes pelos frutos é a seguinte: Pacovan $>$ Mysore $>$ Caju $>$ Prata Anã $>$ Caipira $=$ Caru-Verde $=$ Maçã $=$ Prata $>$ Ouro $>$ Nanica $=$ Terrinha $>$ Caru-Roxa $>$ Nanicão $>$ Prata Graúda $>$ Marmelo. A cultivar Pacovan apresenta maior exportação dos minerais quantificados, enquanto Marmelo exporta menos minerais pelos frutos (Tabela 5). O teor de minerais presente na casca da cultivar Pacovan contribuiu fortemente para a quantidade exportada pelos frutos dessa cultivar. Hoffmann et al. (2010), ao analisar seis cultivares, também constataram maior acúmulo de micronutrientes para Pacovan.

Quanto ao fornecimento de minerais pelas cultivares, para os quais foram calculadas as percentagens de fornecimento, observou-se que a polpa de Caipira e Ouro se destacou no fornecimento de K (Tabela 6). Os maiores percentuais de fornecimento de $\mathrm{P}, \mathrm{Ca}$ e

Tabela 5. Valores médios das quantidades de macro e micronutrientes exportados pelos frutos (base úmida) de 15 cultivares de bananeira (Musa spp. $)^{(1)}$.

\begin{tabular}{|c|c|c|c|c|c|c|c|c|c|c|}
\hline \multirow[t]{2}{*}{ Cultivares } & \multicolumn{5}{|c|}{ Macronutrientes $\left(\mathrm{kg} \mathrm{Mg}^{-1}\right.$ de matéria fresca) } & \multicolumn{4}{|c|}{ Micronutrientes ( $\mathrm{mg} \mathrm{kg}^{-1}$ de matéria fresca) } & \multirow[t]{2}{*}{ Ranqueamento $^{(2)}$} \\
\hline & $\mathrm{N}$ & $\mathrm{P}$ & $\mathrm{K}$ & $\mathrm{Mg}$ & $\mathrm{Ca}$ & $\mathrm{Fe}$ & $\mathrm{Zn}$ & $\mathrm{Mn}$ & $\mathrm{Cu}$ & \\
\hline Ouro & $3,19 b$ & $0,01 \mathrm{c}$ & $3,35 b$ & $0,21 \mathrm{c}$ & $0,08 \mathrm{e}$ & $9,55 \mathrm{a}$ & $7,21 \mathrm{a}$ & $2,15 \mathrm{~d}$ & $1,35 \mathrm{a}$ & 22 \\
\hline Nanica & $3,49 \mathrm{a}$ & $0,01 \mathrm{c}$ & $3,28 b$ & $0,18 \mathrm{c}$ & $0,08 \mathrm{e}$ & $9,31 \mathrm{a}$ & $5,73 b$ & $1,28 \mathrm{~d}$ & $1,11 \mathrm{~b}$ & 23 \\
\hline Nanicão & $3,27 b$ & $0,01 d$ & $3,09 \mathrm{c}$ & $0,22 \mathrm{c}$ & $0,10 \mathrm{~d}$ & $9,49 \mathrm{a}$ & $7,17 \mathrm{a}$ & $2,11 \mathrm{~d}$ & $0,87 \mathrm{c}$ & 25 \\
\hline Caru-Roxa & $2,43 d$ & $0,01 \mathrm{c}$ & $3,25 b$ & $0,21 \mathrm{c}$ & $0,13 \mathrm{c}$ & $7,64 \mathrm{c}$ & $6,54 b$ & $4,09 \mathrm{c}$ & $1,17 \mathrm{a}$ & 24 \\
\hline Caru-Verde & $2,82 \mathrm{c}$ & $0,01 \mathrm{c}$ & $3,56 \mathrm{a}$ & $0,20 \mathrm{c}$ & $0,12 \mathrm{c}$ & $8,74 b$ & $7,19 \mathrm{a}$ & $4,20 \mathrm{c}$ & $1,13 b$ & 21 \\
\hline Caipira & $2,67 d$ & $0,02 b$ & $3,37 \mathrm{a}$ & $0,18 \mathrm{c}$ & $0,13 \mathrm{c}$ & $7,53 \mathrm{c}$ & $6,10 \mathrm{~b}$ & $8,17 b$ & $1,28 \mathrm{a}$ & 21 \\
\hline Prata & $2,72 \mathrm{~d}$ & $0,01 \mathrm{c}$ & $3,17 \mathrm{~b}$ & $0,27 b$ & $0,16 b$ & $8,44 b$ & $7,19 \mathrm{a}$ & $3,95 \mathrm{c}$ & $1,04 \mathrm{~b}$ & 21 \\
\hline Prata Anã & $2,98 \mathrm{c}$ & $0,01 \mathrm{c}$ & $2,90 \mathrm{c}$ & $0,26 b$ & $0,15 b$ & $8,13 b$ & $7,77 \mathrm{a}$ & $4,62 \mathrm{c}$ & $1,48 \mathrm{a}$ & 20 \\
\hline Maçã & $2,96 \mathrm{c}$ & $0,02 \mathrm{a}$ & $3,26 \mathrm{~b}$ & $0,29 a$ & $0,10 \mathrm{~d}$ & $8,10 \mathrm{~b}$ & $6,54 b$ & $2,41 d$ & $1,02 b$ & 21 \\
\hline Mysore & $2,80 \mathrm{c}$ & $0,02 b$ & $3,57 \mathrm{a}$ & $0,29 a$ & $0,17 \mathrm{~b}$ & $10,11 \mathrm{a}$ & $6,10 \mathrm{~b}$ & $4,58 \mathrm{c}$ & $1,10 \mathrm{~b}$ & 17 \\
\hline Pacovan & $2,85 \mathrm{c}$ & $0,01 \mathrm{c}$ & $3,39 b$ & $0,30 \mathrm{a}$ & $0,18 \mathrm{a}$ & $10,09 a$ & $7,98 \mathrm{a}$ & $5,33 \mathrm{c}$ & $1,26 \mathrm{a}$ & 16 \\
\hline Terrinha & $3,64 \mathrm{a}$ & $0,01 \mathrm{c}$ & $3,33 b$ & $0,21 \mathrm{c}$ & $0,05 f$ & $9,53 \mathrm{a}$ & $7,34 \mathrm{a}$ & $5,08 \mathrm{c}$ & $0,79 \mathrm{c}$ & 23 \\
\hline Marmelo & $2,59 \mathrm{~d}$ & $0,02 \mathrm{a}$ & $3,36 \mathrm{~b}$ & $0,23 c$ & $0,07 \mathrm{e}$ & $8,61 \mathrm{~b}$ & $6,54 b$ & $2,87 d$ & $0,93 \mathrm{c}$ & 27 \\
\hline Prata Graúda & $3,07 \mathrm{~b}$ & $0,01 \mathrm{e}$ & $2,66 \mathrm{c}$ & $0,20 \mathrm{c}$ & $0,09 \mathrm{~d}$ & $8,35 b$ & $5,98 b$ & $4,76 \mathrm{c}$ & $1,07 \mathrm{~b}$ & 26 \\
\hline Caju & $2,72 d$ & $0,02 b$ & $3,71 \mathrm{a}$ & $0,21 \mathrm{c}$ & $0,16 b$ & $8,48 b$ & $4,92 \mathrm{~b}$ & $26,50 \mathrm{a}$ & $1,27 \mathrm{a}$ & 18 \\
\hline Média & 2,95 & 0,019 & 3,28 & 0,23 & 0,12 & 8,76 & 6,69 & 5,47 & 1,12 & - \\
\hline $\mathrm{CV}(\%)$ & 5,85 & 5,30 & 5,73 & 6,63 & 9,82 & 5,14 & 9,78 & 19,01 & 12,24 & - \\
\hline
\end{tabular}

${ }^{(1)}$ Médias seguidas de letras iguais, nas colunas, não diferem pelo teste de Scott \& Knott, a $1 \%$ de probabilidade. ${ }^{(2)}$ Ranqueamento das cultivares por nota quanto aos teores de minerais, em que as letras correspondem a cada média: $\mathrm{a}=1, \mathrm{~b}=2, \mathrm{c}=3, \mathrm{~d}=4$, e assim sucessivamente para cálculo da nota geral. 
Tabela 6. Percentagem de minerais fornecidos por $100 \mathrm{~g}$ de polpa e $100 \mathrm{~g}$ de casca frescas das cultivares Caipira, Prata Anã, Nanicão e Ouro, com base na ingestão dietética de referência ${ }^{(1)}$.

\begin{tabular}{|c|c|c|c|c|c|c|c|c|}
\hline \multirow[t]{2}{*}{ Cultivares } & \multicolumn{8}{|c|}{ Fornecimento de minerais $(\%)^{(2)}$} \\
\hline & $\mathrm{K}$ & $\mathrm{P}$ & $\mathrm{Ca}$ & $\mathrm{Mg}$ & $\mathrm{Fe}$ & $\mathrm{Zn}$ & $\mathrm{Mn}$ & $\mathrm{Cu}$ \\
\hline & \multicolumn{8}{|c|}{ Polpa dos frutos } \\
\hline Caipira & $6,04 a$ & $0,37 \mathrm{a}$ & $0,70 \mathrm{a}$ & $7,14 \mathrm{~b}$ & $9,66 \mathrm{~b}$ & $4,77 \mathrm{~b}$ & $20,65 \mathrm{a}$ & $15,62 \mathrm{a}$ \\
\hline Prata Anã & $4,83 \mathrm{~b}$ & $0,24 \mathrm{~b}$ & $0,63 b$ & $8,83 \mathrm{a}$ & $11,39 a$ & $6,37 \mathrm{a}$ & $11,55 b$ & $17,08 \mathrm{a}$ \\
\hline Nanicão & $4,97 b$ & $0,23 b$ & $0,38 \mathrm{c}$ & $8,19 \mathrm{a}$ & $11,20 \mathrm{a}$ & $5,88 \mathrm{a}$ & $6,63 \mathrm{c}$ & $10,55 \mathrm{~b}$ \\
\hline Ouro & $5,45 \mathrm{a}$ & $0,22 b$ & $0,30 \mathrm{~d}$ & $7,40 \mathrm{~b}$ & $11,51 \mathrm{a}$ & $6,50 \mathrm{a}$ & $5,68 \mathrm{c}$ & $16,38 \mathrm{a}$ \\
\hline \multirow[t]{2}{*}{ CV $(\%)$} & 5,97 & 6,43 & 4,58 & 4,03 & 5,32 & 9,71 & 14,03 & 11,48 \\
\hline & \multicolumn{8}{|c|}{ Casca dos frutos } \\
\hline Caipira & $11,33 \mathrm{~b}$ & $0,25 b$ & $2,58 \mathrm{~b}$ & $4,05 \mathrm{c}$ & $4,77 b$ & $5,79 b$ & $57,33 \mathrm{a}$ & $13,40 \mathrm{a}$ \\
\hline Prata Anã & $8,64 \mathrm{c}$ & $0,17 \mathrm{c}$ & $2,92 \mathrm{a}$ & $6,83 \mathrm{a}$ & $6,37 \mathrm{a}$ & $7,05 \mathrm{a}$ & $30,35 b$ & $13,96 \mathrm{a}$ \\
\hline Nanicão & $12,05 b$ & $0,19 \mathrm{c}$ & $2,23 b$ & $5,06 \mathrm{~b}$ & $5,88 \mathrm{a}$ & $6,71 \mathrm{a}$ & $14,45 \mathrm{c}$ & $7,91 \mathrm{~b}$ \\
\hline Ouro & $16,52 \mathrm{a}$ & $0,28 \mathrm{a}$ & $2,93 \mathrm{a}$ & $5,55 \mathrm{~b}$ & $6,50 \mathrm{a}$ & $7,41 \mathrm{a}$ & $20,65 \mathrm{c}$ & $14,44 \mathrm{a}$ \\
\hline CV (\%) & 4,46 & 6,32 & 7,51 & 5,56 & 9,71 & 8,21 & 10,31 & 8,38 \\
\hline
\end{tabular}

${ }^{(1)}$ Médias seguidas de letras iguais, nas colunas, para polpa e casca não diferem pelo teste de Scott \& Knott, a $1 \%$ de probabilidade. ${ }^{(2)}$ Fornecimento de minerais com base na ingestão dietética de referência para homens adultos (National Research Council, 2001, 2004).

Mn também foram obtidos com o consumo da polpa da cultivar Caipira. Entretanto, para Fe e Zn, a cultivar Caipira foi a que menos contribuiu. Na casca, a cultivar Ouro foi a que mais forneceu K e P. Para Mg e Mn, o maior fornecimento foi das cultivares Prata Anã e Caipira, respectivamente. Para os outros minerais, a percentagem variou entre as cultivares. Wall (2006), ao estudar a polpa madura das cultivares Nanica e Williams, obteve percentagem de fornecimento de K, $\mathrm{P}, \mathrm{Mn}$ e $\mathrm{Cu}$ superior à encontrada no presente trabalho. Para $\mathrm{Ca}$ e $\mathrm{Mg}$, as percentagens foram próximas. Já para Fe e Zn, a percentagem observada pelo autor foi menor.

Cabe ressaltar que os cálculos realizados não levaram em conta a biodisponibilidade para o ser humano, a qual é afetada por diversos fatores, como a presença de inibidores ou compostos com ação sinérgica no alimento, a solubilidade, a concentração e o estado de oxidação do mineral (Davey et al., 2009).

\section{Conclusões}

1. Há diferenças entre cultivares de bananeiras (Musa spp.) quanto à concentração de macro e micronutrientes na casca e na polpa, mas não entre frutos verdes e maduros.

2. A polpa da cultivar Caipira (AAA) e a casca das cultivares Prata e Pacovan (AAB) apresentam os maiores teores de minerais.
3. A ordem de absorção e exportação dos nutrientes minerais pelos frutos é $\mathrm{K}>\mathrm{N}>\mathrm{Mg}>\mathrm{Ca}>\mathrm{P}>\mathrm{Fe}>\mathrm{Zn}$ $>\mathrm{Mn}>\mathrm{Cu}$.

4. A polpa da cultivar Caipira e a casca da cultivar Ouro apresentam maior capacidade de fornecimento de minerais, com base na ingestão dietética de referência.

\section{Agradecimento}

Ao Conselho Nacional de Desenvolvimento Científico e Tecnológico (CNPq), pelo apoio financeiro e pela concessão de bolsas de estudo e de produtividade em pesquisa.

\section{Referências}

AGAMA-ACEVEDO, E.; ISLAS-HERNÁNDEZ, J.J.; PACHECO-VARGAS, G.; OSORIO-DIAZ, P.; BELLO-PÉREZ, L.A. Starch digestibility and glycemic index of cookies partially substituted with unripe banana flour. LWT - Food Science and Technology, v.46, p.177-182, 2012.

ALKARKHI, A.F.M.; BIN RAMLI, S.; YONG, Y.S.; EASA, A.M. Comparing physicochemical properties of banana pulp and peel flours prepared from green and ripe fruits. Food Chemistry, v.129, p.312-318, 2011. DOI: 10.1016/j.foodchem.2011.04.060.

APARICIO-SAGUILAN, A.; SAYAGO-AYERDI, S.G.; VARGAS-TORRES, A.; JUSCELINO, T.; ASCENCIO-OTERO, T.E.; BELLO-PEREZ, L.A. Slowly digestible cookies prepared from resistant starch-rich lintnerized banana starch. Journal of Food Composition and Analysis, v.20, p.175-181, 2007. DOI: 10.1016/j.jfca.2006.07.005. 
BRAGA, J.M.;DEFELIPO, B.V. Determinação espectrofotométrica de $\mathrm{P}$ em extratos de solo e material vegetal. Revista Ceres, v.21, p.73-85, 1974.

DADZIE, B.K.; ORCHARD, J.E. Evaluación rutinaria postcosecha de híbridos de bananos y plátanos: criterios y métodos. Montpelier: INIBAP, 1997. 63p. (Inibap. Guias técnicas, 2).

DAVEY, M.W.; VAN DEN BERGH, I.; MARKHAM, R.; SWNNEN, R.; KEULEMANS, J. Genetic variability in Musa fruit provitamin A carotenoids, lutein and mineral micronutrient contents. Food Chemistry, v.115, p.806-813, 2009. DOI: 10.1016/j.foodchem.2008.12.088.

DAVEY, M.W.; STALS, E.; NGOH-NEWILAH, G.; TOMEKPE, K.; LUSTY, C.; MARKHAM, R.; SWENNEN, R.; KEULEMANS, J. Sampling strategies and variability in fruit pulp micronutrient contents of West and Central African bananas and plantains (Musa species). Journal of Agricultural and Food Chemistry, v.55, p.2633-2644, 2007. DOI: 10.1021/jf0631191.

EMAGA, T.H.; ANDRIANAIVO, R.H.; WATHELET, B.; TCHANGO, J.T.; PAQUOT, M. Effects of the stage of maturation and varieties on the chemical composition of banana and plantain peels. Food Chemistry, v.103, p.590-600, 2007. DOI: 10.1016/j. foodchem.2006.09.006.

FOOD AND AGRICULTURE ORGANIZATION OF THE UNITED NATIONS. FaoStat. Available at: $<$ http://faostat.fao.org/ site/339; defaut.aspx>. Accessed on: 22 fev. 2014.

GOMES, M. da C.; VIANA, A.P.; OLIVEIRA, J.G. de; PEREIRA, M.G.; GONCALVES, G.M.; FERREIRA, C.F. Avaliação de germoplasma elite de bananeira. Revista Ceres, v.54, p.185-190, 2007.

GONDIM, J.A.M.; MOURA, M. de F.V.; DANTAS, A.S.; MEDEIROS, R.L.S.; SANTOS, S.M. Composição centesimal e de minerais em cascas de frutas. Ciência e Tecnologia de Alimentos, v.25, p.825-827, 2005. DOI: 10.1590/S0101-20612005000400032.

HELRICH, K. Official methods of analysis of the Association of Official Analytical Chemists. $15^{\text {th }}$ ed. Arlington: Association of Official Analytical Chemists, 1990. 2v.

HOFFMANN, R.B.; OLIVEIRA, F.H.T. de; GHEYI, H.R.; SOUZA, A.P. de; ARRUDA, J.A. de. Acúmulo de matéria seca, absorção e exportação de micronutrientes em variedades de bananeira sob irrigação. Ciência e Agrotecnologia, v.34, p.536-544, 2010. DOI: 10.1590/S1413-70542010000300002.
JUAREZ-GARCIA, E.; AGAMA-ACEVEDO, E.; SAYAGO-AYERDI, S.G.; RODRÍGUEZ-AMBRIZ， S.L.; BELLO-PÉREZ, L.A. Composition, digestibility and application in breadmaking of banana flour. Plant Foods for Human Nutrition, v.61, p.131-137, 2006. DOI: 10.1007/s11130-006-0020-x.

KALEMELAWA, F.; NISHIHARA, E.; TSUNEYOSHI, E.; AHMAD, Z.; YEASMI, R.; TENYWA, M.M.; YAMAMOTO, S. An evaluation of aerobic and anaerobic composting of banana peels treated with different inoculums for soil nutrient replenishment. Bioresource Technology, v.126, p.375-382, 2012. DOI: 10.1016/j. biortech.2012.04.030.

NATIONAL RESEARCH COUNCIL. Dietary reference intakes for water, potassium, sodium, chloride, and sulfate. Washington: The National Academy Press, 2004.

NATIONAL RESEARCH COUNCIL. Dietary reference intakes for vitamin A, vitamin $K$, arsenic, boron, chromium, copper, iodine, iron, manganese, molybdenum, nickel, silicon, vanadium, and zinc. Washington: The National Academy Press, 2001.

RAMOS, D.P.; LEONEL, S.; MISCHAN, M.M. Caracterização físico-química dos frutos de genótipos de bananeira produzidos em Botucatu-SP. Ciência e Agrotecnologia, v.33, p.1765-1770, 2009. DOI: $10.1590 /$ S1413-70542009000700011.

SAEG: sistema para análises estatísticas. Versão 9.1. Viçosa: Fundação Arthur Bernardes/UFV, 2007. 301p.

SOARES, F.A.L.; GHEYI, H.R.; OLIVEIRA, F.H.T. de; FERNANDES, P.D.; ALVES, A.N.; SILVA, F.V. da. Acúmulo, exportação e restituição de nutrientes pelas bananeiras 'Prata Anã' e 'Grand Naine'. Ciência Rural, v.38, p.2054-2058, 2008. DOI: 10.1590/S0103-84782008000700042.

SOTHORNVIT,R.; PITAK, N.Oxygen permeability andmechanical properties of banana films. Food Research International, v.40, p.365-370, 2007. DOI: 10.1016/j.foodres.2006.10.010.

TEIXEIRA, L.A.J.; VAN RAIJ, B.; BETTIOL NETO, J.E. Estimativa das necessidades nutricionais de bananeiras do subgrupo Cavendish cultivadas no Estado de São Paulo. Revista Brasileira de Fruticultura, v.30, p.540-545, 2008. DOI: 10.1590/ S0100-29452008000200047.

WALL, M.M. Ascorbic acid, vitamin A, and mineral composition of banana (Musa sp.) and papaya (Carica papaya) cultivars grown in Hawaii. Journal of Food Composition and Analysis, v.19, p.434-445, 2006. DOI: 10.1016/j.jfca.2006.01.002.

Recebido em 7 de março de 2014 e aprovado em 10 de julho de 2014 